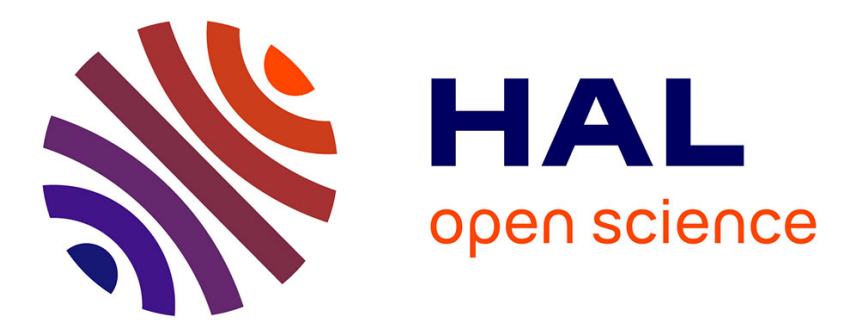

\title{
Données récentes sur la physiopathologie et les approches thérapeutiques des lymphomes T cutanés épidermotropes
}

M. Bagot

\section{- To cite this version:}

M. Bagot. Données récentes sur la physiopathologie et les approches thérapeutiques des lymphomes T cutanés épidermotropes. Bulletin de l'Académie Nationale de Médecine, 2019, 203, pp.701 - 705. 10.1016/j.banm.2019.10.003 . hal-03488358

\section{HAL Id: hal-03488358 \\ https://hal.science/hal-03488358}

Submitted on 21 Dec 2021

HAL is a multi-disciplinary open access archive for the deposit and dissemination of scientific research documents, whether they are published or not. The documents may come from teaching and research institutions in France or abroad, or from public or private research centers.
L'archive ouverte pluridisciplinaire HAL, est destinée au dépôt et à la diffusion de documents scientifiques de niveau recherche, publiés ou non, émanant des établissements d'enseignement et de recherche français ou étrangers, des laboratoires publics ou privés.

\section{(ㄷ)(1) $\$$}

Distributed under a Creative Commons Attribution - NonCommercial| 4.0 International 
BANM 203/8-9

\section{REVUE GÉNÉRALE}

Reçu le 20 septembre 2018

Accepté le 18 mars 2019

\section{Données récentes sur la physiopathologie et les approches thérapeutiques des lymphomes $\mathbf{T}$ cutanés épidermotropes}

\section{Recent progress in the pathophysiology and the treatment of epidermotropic cutaneous $T$ cell lymphoma}

\section{Martine Bagot}

Service de Dermatologie, APHP, Hôpital Saint Louis, Université Paris 7, Unité Inserm U976, 1 Avenue Claude Vellefaux, 75010 Paris

martine.bagot@aphp.fr

Liens d'intérêt : Martine Bagot est co-auteur du brevet et membre du Comité Scientifique Innate Pharma pour le développement d'IPH4102 pour le traitement des lymphomes T cutanés

Mots clés : Lymphome T cutané ; Mycosis fongoïde ; Syndrome de Sézary ; biothérapie ; anticorps monoclonal

Key words : Cutaneous T cell lymphoma ; Mycosis fungoides ; Sezary syndrome ; biotherapy ; monoclonal antibody

*Séance du 25 juin 2019 


\title{
Résumé
}

Les lymphomes T cutanés représentent un groupe hétérogène de lymphomes rares débutant et prédominant au niveau de la peau. Les plus fréquents sont le mycosis fongoïde et le syndrome de Sézary. Il n'existe malheureusement pas actuellement de traitement curatif de ces lymphomes T cutanés épidermotropes aux stades avancés. IPH4102 est un nouvel anticorps monoclonal humanisé dirigé contre le récepteur natural killer KIR3DL2/CD158k. KIR3DL2 est exprimé par les lymphocytes tumoraux de $85 \%$ des lymphomes T cutanés, en particulier les syndromes de Sézary et les mycosis fongoïdes au stade avancé. La tolérance et l'efficacité de cette biothérapie a été étudiée dans un essai multicentrique international "first in human » de phase I/II. Les malades inclus étaient des lymphomes $\mathrm{T}$ cutanés en rechute ou réfractaires après au moins deux lignes de traitements systémiques, et exprimant KIR3DL2 dans la peau et/ou le sang. Cette étude a montré la très bonne tolérance de cet anticorps ciblé, et des résultats d'efficacité très prometteurs, particulièrement chez les malades ayant un syndrome de Sézary.

\begin{abstract}
Cutaneous $\mathrm{T}$ cell lymphomas represent a heterogenous group of rare lymphomas beginning and predominating in the skin. Mycosis fungoides and Sezary syndrome are the most frequent subgroups of patients. There is currently no curative treatment for these advanced stage epidermotropic cutaneous $\mathrm{T}$ cell lymphomas. IPH4102 is a novel humanized monoclonal antibody specific for the natural killer receptor KIR3DL2/CD158k. KIR3DL2 is expressed by tumor T lymphocytes of $85 \%$ of cutaneous $\mathrm{T}$ cell lymphomas, especially Sezary syndrome, and advanced mycosis fungoides. Tolerance and efficacy of this new biotherapy have been evaluated in a multicentric international "first in human" phase I/II trial. Patients included were patients with a cutaneous T-cell lymphoma having failed at least two prior lines of systemic therapy and expressing KIR3DL2 in skin and/or blood. This trial demonstrated the very good tolerance of this targeted biotherapy. Efficacy was also very promising, especially in the group of patients with a Sezary syndrome.
\end{abstract}


Les lymphomes T cutanés représentent un groupe hétérogène de lymphomes rares débutant et predominant au niveau de la peau. Les plus fréquents sont le mycosis fongoïde et le syndrome de Sézary. Le mycosis fongoïde est caractérisé par des plaques érythémateuses prurigineuses pouvant secondairement s'infiltrer et s'associer à des tumeurs. Le syndrome de Sézary réalise une érythrodermie souvent associée à des adénopathies, avec détection dans le sang et la peau d'une prolifération monoclonale de lymphocytes $\mathrm{T}[1,2]$. Aux stades précoces, le mycosis fongoïde a une évolution indolente et relève de traitements locaux [3]. En revanche, aux stades avancés, ces lymphomes épidermotropes ont une évolution plus défavorable en raison de l'évolution progressive des lésions cutanées et ganglionnaires et de la fréquence des complications infectieuses. La survie moyenne des patients au stade de tumeurs varie de 4 à 6 ans, celle des patients ayant un syndrome de Sézary varie de 2 à 5 ans [4,5]. La majorité des patients à ce stade reçoit de nombreuses lignes successives de traitements induisant le plus souvent des rémissions partielles. Il n'existe malheureusement pas actuellement de traitement curatif de ces lymphomes T cutanés aux stades avancés.

\section{Traitement des lymphomes $T$ cutanés épidermotropes avancés}

Les traitements systémiques des lymphomes $\mathrm{T}$ cutanés épidermotropes avancés comprennent des traitements immunomodulateurs (interféron alpha, bexarotène, méthotrexate), des traitements de radiothérapie (radiothérapie conventionnelle, électronthérapie superficielle totale), ou des photophérèses pour les lymphomes érythrodermiques. Les traitements épigénétiques comme les inhibiteurs d'histone déacétylases (vorinostat, romidepsine), disponibles aux États-Unis, n'ont pas été approuvés en Europe dans cette indication. En cas de maladie évolutive, les malades reçoivent des monochimiothérapies telles que la doxorubicine liposomée ou la gemcitabine, ou différents protocoles de chimiothérapies. Ces traitements induisent le plus souvent des réponses partielles et transitoires [6]. Chez certains malades, il est possible d'obtenir des rémissions complètes prolongées en réalisant des greffes de moëlle allogéniques [7].

\section{Les nouveaux anticorps monoclonaux}

Les traitements par anticorps monoclonaux représentent un nouvel espoir de traitement pour les lymphomes T cutanés.

L'alemtuzumab est un anticorps monoclonal humanisé dirigé contre CD52, un marqueur panlymphocytaire. Cet anticorps a prouvé son efficacité dans les mycosis fongoïdes et les syndromes de Sézary non transformés au prix d'une destruction de la majorité des lymphocytes $\mathrm{T}$ et $\mathrm{B}$, induisant des déficits immunitaires sévères prolongés et des infections parfois fatales $[8,9]$. Des protocoles d'administration de plus faibles doses ont été proposés, qui induisent également des immunodépressions sévères [10].

Le Brentuximab vedotin (SGN-35) (BV) est un anticorps monoclonal humanisé dirigé contre la molécule CD30 et couplé à la monométhyl auristatine [11]. L'efficacité de cet anticorps dans les lymphomes T cutanés épidermotropes (mycosis fongoïde, syndrome de Sézary) a été démontrée dans des études de phase II et III [12-14]. L'étude récente de phase III ALCANZA a démontré la supériorité du BV sur le méthotrexate ou le bexarotène. Le BV a en effet induit une réponse globale durant au moins 4 mois chez 56,3\% des patients traités versus 12,5\% des malades traités par méthotrexate ou bexarotène. La survie médiane sans événement était de 9,4 mois dans le groupe BV versus 2,3 mois dans le groupe méthotrexate ou bexarotène $(\mathrm{p}<0.0001)$. La survie médiane sans progression était de 15,9 mois dans le groupe mycosis 
fongoïde, versus 3,5 mois dans le bras contrôle [14]. Les syndromes de Sézary étaient exclus de cette étude. L'effet secondaire le plus fréquent était la survenue de neuropathies périphériques souvent invalidantes, de régression parfois lente, limitant les possibilités de traitements prolongés.

Le Mogamulizumab (KW-0761) est un anticorps monoclonal humanisé dirigé contre le CC chemokine récepteur 4 (CCR4) [15], exprimé sélectivement par les lymphocytes T à tropisme cutané, les lymphomes T cutanés et les lymphocytes T régulateurs [16,17]. Son efficacité a été démontrée dans des études de phase I/II chez des malades ayant un mycosis fongoïde ou un syndrome de Sézary [18,19]. L'étude randomisée multicentrique de phase III MAVORIC a comparé l'efficacité du mogamulizumab et du vorinostat sur une cohorte de 372 malades ayant un mycosis fongoïde ou un syndrome de Sézary non transformé en rechute ou réfractaire après au moins une ligne de traitement systémique [20]. Cette étude a montré des taux de réponses supérieurs dans le groupe mogamulizumab chez les malades ayant un mycosis fongoïde ( $21 \%$ versus $7,1 \% ; \mathrm{p}=0.0042$ ) ou un syndrome de Sézary ( $37 \%$ versus $2,3 \% ; \mathrm{p}<0.0001)$. La survie médiane sans progression était de 7,7 mois dans le groupe mogamulizumab versus 3,1 mois dans le groupe vorinostat $(\mathrm{p}<0,0001)$. Le traitement était le plus souvent bien toléré mais certains malades ont présenté des éruptions cutanées et des réactions d'auto-immunité.

Malgré leur efficacité tous ces anticorps induisent des réponses partielles et transitoires. De plus, certains effets secondaires de ces anticorps soulignent la nécessité de développer des traitements plus ciblés aux lymphocytes T tumoraux.

\section{IPH4102, un nouvel anticorps anti-KIR3DL2}

IPH4102 est un anticorps monoclonal humanisé dirigé contre le récepteur NK KIR3DL2/CD158k [21]. Cet anticorps se lie à un épitope particulier de la molécule KIR3DL2 sur le domaine immunoglobulinique D0 qui n'entraîne pas l'internalisation du récepteur. La persistance du complexe anticorps-récepteur à la surface des cellules ciblées permet l'induction de fonctions effectrices optimales. KIR3DL2 appartient à la famille des récepteurs KIRs (Killer Inhibitory Receptors) de type inhibiteur. Ce récepteur est exprimé sur une minorité de lymphocytes NK et $\mathrm{CD} 3+\mathrm{CD} 8+[22,23]$. De manière très intéressante, KIR3DL2 est sélectivement exprimé par les lymphocytes tumoraux circulants des patients ayant un syndrome de Sézary [24,28]. Pour le syndrome de Sézary, les études uni- et multivariées ont montré que KIR3DL2 est le meilleur marqueur pour le diagnostic de la maladie mais aussi pour le pronostic [29,30]. En effet, les malades dont $85 \%$ des lymphocytes expriment KR3DL2 ont une survie moyenne de 5,7 ans, alors que les malades dont plus de $85 \%$ des lymphocytes expriment KIR3DL2 ont une survie moyenne de 3,2 ans $(\mathrm{p}=0.0021)$ [30]. Dans la peau, KIR3DL2 est exprimé par les lymphocytes tumoraux de 80\% des lymphomes T cutanés, en particulier les syndromes de Sézary, les mycosis fongoïdes au stade avancé, et les lymphomes $\mathrm{T}$ anaplasiques CD30 positifs [31-33]. KIR3DL2 est également exprimé par la majorité des lymphomes T cutanés associés au virus HTLV-1 et l'intensité d'expression est corrélée au pronostic [34]. KIR3DL2 est donc une cible idéale pour le développement d'anticorps monoclonaux sélectifs.

La signification physiopathologique de l'expression atypique de KIR3DL2 par les lymphocytes $\mathrm{T}$ de lymphomes $\mathrm{T}$ cutanés reste mal comprise. La liaison de l'anticorps au récepteur sur les lymphocytes $\mathrm{T}$ tumoraux n'induit pas de signalisation intra-cellulaire, bien 
que la co-ligation du récepteur avec un anticorps anti-CD3 $\varepsilon$ induise une inhibition de la phosphorylation CD3-induite, suggérant que l'expression atypique de KIR3DL2 pourrait avoir une fonction inhibitrice. Cette co-ligation induit en outre une diminution de la mort cellulaire induite par l'activation, suggérant que ce récepteur pourrait avoir un effet antiapoptotique pour le clone tumoral $[35,36]$.

L'anticorps anti-KIR3DL2 IPH4102 a été développé par le laboratoire Innate Pharma pour le traitement des lymphomes $\mathrm{T}$ cutanés [21]. Les études précliniques ont montré que cet anticorps pouvait induire une lyse et une phagocytose dépendante de l'anticorps des cellules tumorales dans un système autologue. Un modèle murin de xénogreffe de lymphome $\mathrm{T}$ cutané a été construit utilisant des souris CB17 présentant un déficit immunitaire recevant des injections de cellules Raji transfectées par KIR3DL2 par voie intra-veineuse ou sous-cutanée. L'injection de l'anticorps IPH4102 aux souris pouvait induire une prolongation de la survie des souris et une diminution de la taille des tumeurs sous-cutanées.

\section{Étude multicentrique internationale de phase I/II}

La tolérance et l'efficacité de l'anticorps IPH4102 ont été étudiées dans un essai multicentrique international "first in human» de phase I/II associant 3 centres US et 3 centres européens. Les malades inclus étaient des lymphomes $\mathrm{T}$ cutanés en rechute ou réfractaires après au moins 2 lignes de traitements systémiques, et exprimant KIR3DL2 dans la peau et/ou le sang. L'étude comportait 10 escalades de doses, de $0,0001 \mathrm{mg} / \mathrm{kg}$ à $10 \mathrm{mg} / \mathrm{kg}$. Les malades recevaient une injection hebdomadaire les 4 premières semaines, puis toutes les 2 semaines les 20 semaines suivantes, puis tous les mois. Le traitement était poursuivi jusqu'à progression de la maladie ou survenue d'un événement indésirable sévère. 25 malades ont été inclus dans cette phase d'escalade de dose, dont 20 syndromes de Sézary, et 4 mycosis fongoïdes. Ces malades avaient reçu une moyenne de 4 (2 à 10) traitements précédents. Le traitement a été très bien toléré, puisqu'aucune toxicité limitant la dose n'a été observée jusqu'à la posologie de $10 \mathrm{mg} / \mathrm{kg}$. Une «flat dose » de $750 \mathrm{mg}$ a été en conséquence choisie pour la phase d'extension.

La tolérance de ce traitement a été très bonne et il n'a pas été observé d'augmentation de fréquence des infections ni de phénomènes d'auto-immunité [38]. L'efficacité de l'anticorps IPH4102 a été évaluée selon les critères internationaux en particulier pour la peau le mSWAT (modified severity weighted assessment tool). $44 \%$ des malades et $50 \%$ des Sézary ont présenté une réponse globale complète ou partielle (diminution de plus de $50 \%$ des lésions). Parmi les patients ayant un syndrome de Sézary, $60 \%$ ont présenté une réponse dans la peau et $65 \%$ une réponse au niveau du sang. Les scores de prurit et de qualité de vie étaient très améliorés chez les malades répondeurs mais également chez les malades ayant une maladie stable [37-38].

Les études translationnelles ont montré une diminution parallèle du clone tumoral et des lymphocytes CD4+CD158k+ dans le sang, corrélée à la réponse clinique. La diminution des lymphocytes CD158k+ dans la peau était également corrélée à la réponse clinique et des taux plus élevés de lymphocytes CD8 dans la peau étaient associés à une meilleure réponse. Les études en biologie moléculaires ont montré chez les malades en rémission complète une disparition totale et prolongée du clone $\mathrm{T}$ tumoral dans la peau et le sang. Le nombre et la fonction des lymphocytes NK du sang sont restés normaux pendant toute la durée de l'étude [39]. 


\section{Conclusion}

L'anticorps IPH4102 est donc une nouvelle biothérapie ciblée dont la tolérance et l'efficacité sont très prometteurs pour le traitement sélectif des malades ayant un lymphome $\mathrm{T}$ cutané épidermotrope. Des études d'extension sont en cours afin de valider l'intérêt particulier de cet anticorps pour les malades ayant un syndrome de Sézary. 


\section{Références}

[1] Olsen E, Vonderheid E, Pimpinelli N, Willemze R, Kim Y, Knobler R, et al. Revisions to the staging and classification of mycosis fungoides and Sezary syndrome: a proposal of the International Society for Cutaneous Lymphomas (ISCL) and the cutaneous lymphoma task force of the European Organization of Research and Treatment of Cancer (EORTC). Blood. 2007;110:1713-22.

[2] Bradford PT, Devesa SS, Anderson WF, Toro JR. Cutaneous lymphoma incidence patterns in the United States: a population-based study of 3884 cases. Blood. 2009;113:5064-73.

[3] Trautinger F, Eder J, Assaf C, Bagot M, Cozzio A, Dummer R, et al. European Organisation for Research and Treatment of Cancer consensus recommendations for the treatment of mycosis fungoides/Sézary syndrome - Update 2017. Eur J Cancer. 2017;77:57-74.

[4] Agar NS, Wedgeworth E, Crichton S, Mitchell TJ, Cox M, Ferreira S, et al. Survival outcomes and prognostic factors in mycosis fungoides/Sezary syndrome: validation of the revised International Society for Cutaneous Lymphomas/European Organisation for Research and Treatment of Cancer staging proposal. J Clin Oncol. 2010;28:4730-9.

[5] Talpur R, Singh L, Daulat S, Liu P, Seyfer S, Trynosky T, et al. Long-term outcomes of 1,263 patients with mycosis fungoides and Sezary syndrome from 1982 to 2009. Clin Cancer Res. 2012;18:5051-60.

[6] Hughes CF, Khot A, McCormack C, Lade S, Westerman DA, Twigger R, et al. Lack of durable disease control with chemotherapy for mycosis fungoides and Sezary syndrome: a comparative study of systemic therapy. Blood. 2015;125:71-81.

[7] de Masson A, Beylot-Barry M, Bouaziz JD, Peffault de Latour R, Aubin F, Garciaz S, et al. Allogeneic stem cell transplantation for advanced cutaneous T-cell lymphomas: a study from the French Society of Bone Marrow Transplantation and French Study Group on Cutaneous Lymphomas. Haematologica. 2014;99:527-34.

[8] Querfeld C, Mehta N, Rosen ST, Guitart J, Rademaker A, Gerami P, et al. Alemtuzumab for relapsed and refractory erythrodermic cutaneous T-cell lymphoma: a single institution experience from the Robert H. Lurie Comprehensive Cancer Center. Leuk Lymphoma. 2009;50:1969-76.

[9] de Masson A, Guitera P, Brice P, Moulonguet I, Mouly F, Bouaziz JD, et al. Long-term efficacy and safety of alemtuzumab in advanced primary cutaneous T-cell lymphomas. Br J Dermatol. 2014;170:720-4.

[10] Bernengo MG, Quaglino P, Comessatti A, Ortoncelli M, Novelli M, Lisa F, et al. Lowdose intermittent alemtuzumab in the treatment of Sezary syndrome: clinical and immunologic findings in 14 patients. Haematologica. 2007;92:784-94.

[11] Francisco JA, Cerveny CG, Meyer DL, Mixan BJ, Klussman K, Chace DF, et al. cAC10vcMMAE, an anti-CD30-monomethyl auristatin E conjugate with potent and selective antitumor activity. Blood. 2003;102:1458-65.

[12] Kim YH, Tavallaee M, Sundram U, Salva KA, Wood GS, Li S, et al. Phase II Investigator-Initiated Study of Brentuximab Vedotin in Mycosis Fungoides and Sezary Syndrome With Variable CD30 Expression Level: A Multi-Institution Collaborative Project. J Clin Oncol. 2015;33:3750-8.

[13] Duvic M, Tetzlaff MT, Gangar P, Clos AL, Sui D, Talpur R. Results of a Phase II Trial of Brentuximab Vedotin for CD30+ Cutaneous T-Cell Lymphoma and Lymphomatoid Papulosis. J Clin Oncol. 2015;33:3759-65.

[14] Prince HM, Kim YH, Horwitz SM, Dummer R, Scarisbrick J, Quaglino P, et al. Brentuximab vedotin or physician's choice in CD30-positive cutaneous T-cell 
lymphoma (ALCANZA): an international, open-label, randomised, phase 3, multicentre trial. Lancet. 2017;390:555-66.

[15] Yamamoto K, Utsunomiya A, Tobinai K, Tsukasaki K, Uike N, Uozumi K, et al. Phase I study of KW-0761, a defucosylated humanized anti-CCR4 antibody, in relapsed patients with adult T-cell leukemia-lymphoma and peripheral T-cell lymphoma. J Clin Oncol. 2010;28:1591-8.

[16] Imai T, Nagira M, Takagi S, Kakizaki M, Nishimura M, Wang J, et al. Selective recruitment of CCR4-bearing Th2 cells toward antigen-presenting cells by the CC chemokines thymus and activation-regulated chemokine and macrophage-derived chemokine. Int Immunol. 1999;11:81-8.

[17] Ishida T, Utsunomiya A, Iida S, Inagaki H, Takatsuka Y, Kusumoto S, et al. Clinical significance of CCR4 expression in adult T-cell leukemia/lymphoma: its close association with skin involvement and unfavorable outcome. Clin Cancer Res. 2003;9(10 Pt 1):3625-34.

[18] Ogura M, Ishida T, Hatake K, Taniwaki M, Ando K, Tobinai K, et al. Multicenter phase II study of mogamulizumab (KW-0761), a defucosylated anti-cc chemokine receptor 4 antibody, in patients with relapsed peripheral T-cell lymphoma and cutaneous T-cell lymphoma. J Clin Oncol. 2014;32:1157-63.

[19] Duvic M, Pinter-Brown LC, Foss FM, Sokol L, Jorgensen JL, Challagundla P, et al. Phase 1/2 study of mogamulizumab, a defucosylated anti-CCR4 antibody, in previously treated patients with cutaneous T-cell lymphoma. Blood. 2015;125:1883-9.

[20] Kim YH, Bagot M, Pinter-Brown L, Rook AH, Porcu P, Horwitz SM, et al. Anti-CCR4 monoclonal antibody, mogamulizumab, demonstrates significant improvement in PFS compared to vorinostat in patients with previously treated cutaneous T-cell lymphoma (CTCL): results from the phase III MAVORIC study. Blood. 2017;130(Supplement 1):817.

[21] Marie-Cardine A, Viaud N, Thonnart N, Joly R, Chanteux S, Gauthier L, et al. IPH4102, a humanized KIR3DL2 antibody with potent activity against cutaneous T-cell lymphoma. Cancer Res. 2014;74:6060-70.

[22] Moretta A, Biassoni R, Bottino C, Pende D, Vitale M, Poggi A, et al. Major histocompatibility complex class I-specific receptors on human natural killer and $\mathrm{T}$ lymphocytes. Immunol Rev. 1997;155:105-17.

[23] Chan AT, Kollnberger SD, Wedderburn LR, Bowness P. Expansion and enhanced survival of natural killer cells expressing the killer immunoglobulin-like receptor KIR3DL2 in spondylarthritis. Arthritis Rheum. 2005;52:3586-95.

[24] Bagot M, Moretta A, Sivori S, Biassoni R, Cantoni C, Bottino C, et al. CD4(+) cutaneous T-cell lymphoma cells express the p140-killer cell immunoglobulin-like receptor. Blood. 2001;97:1388-91.

[25] Poszepczynska-Guigne E, Schiavon V, D'Incan M, Echchakir H, Musette P, Ortonne N, et al. CD158k/KIR3DL2 is a new phenotypic marker of Sezary cells: relevance for the diagnosis and follow-up of Sezary syndrome. J Invest Dermatol. 2004;122:820-3.

[26] Ortonne N, Huet D, Gaudez C, Marie-Cardine A, Schiavon V, Bagot M, et al. Significance of circulating T-cell clones in Sezary syndrome. Blood. 2006;107:4030-8.

[27] Bahler DW, Hartung L, Hill S, Bowen GM, Vonderheid EC. CD158k/KIR3DL2 is a useful marker for identifying neoplastic T-cells in Sezary syndrome by flow cytometry. Cytometry B Clin Cytom. 2008;74:156-62.

[28] Bouaziz JD, Remtoula N, Bensussan A, Marie-Cardine A, Bagot M. Absolute CD3+ CD158k+ lymphocyte count is reliable and more sensitive than cytomorphology to evaluate blood tumour burden in Sezary syndrome. Br J Dermatol. 2010;162:123-8. 
[29] Moins-Teisserenc H, Daubord M, Clave E, Douay C, Felix J, Marie-Cardine A, et al. $\mathrm{CD} 158 \mathrm{k}$ is a reliable marker for diagnosis of Sezary syndrome and reveals an unprecedented heterogeneity of circulating malignant cells. J Invest Dermatol. 2015;135:247-57.

[30] Hurabielle C, Thonnart N, Ram-Wolff C, Sicard H, Bensussan A, Bagot M, et al. Usefulness of KIR3DL2 to Diagnose, Follow-Up, and Manage the Treatment of Patients with Sezary Syndrome. Clin Cancer Res. 2017;23:3619-27.

[31] Ortonne N, Le Gouvello S, Tabak R, Marie-Cardine A, Setiao J, Berrehar F, et al. CD158k/KIR3DL2 and NKp46 are frequently expressed in transformed mycosis fungoides. Exp Dermatol. 2012;21:461-3.

[32] Battistella M, Janin A, Jean-Louis F, Collomb C, Leboeuf C, Sicard H, et al. KIR3DL2 $(\mathrm{CD} 158 \mathrm{k})$ is a potential therapeutic target in primary cutaneous anaplastic large-cell lymphoma. Br J Dermatol. 2016;175:325-33.

[33] Battistella M, Leboeuf C, Ram-Wolff C, Hurabielle C, Bonnafous C, Sicard H, et al. KIR3DL2 expression in cutaneous T-cell lymphomas: expanding the spectrum for KIR3DL2 targeting. Blood. 2017;130:2900-2902.

[34] Hurabielle C, Leboeuf C, Ram-Wolff C, Meignin V, Rivet J, Vignon-Pennamen MD, et al. KIR3DL2 expression in patients with adult T-cell lymphoma/leukaemia. $\mathrm{Br} \mathrm{J}$ Dermatol. 2018, [en ligne].

[35] Thonnart N, Caudron A, Legaz I, Bagot M, Bensussan A, Marie-Cardine A. KIR3DL2 is a coinhibitory receptor on Sezary syndrome malignant $\mathrm{T}$ cells that promotes resistance to activation-induced cell death. Blood. 2014;124:3330-2.

[36] Ghazi B, Thonnart N, Bagot M, Bensussan A, Marie-Cardine A. KIR3DL2/CpG ODN interaction mediates Sezary syndrome malignant $\mathrm{T}$ cell apoptosis. J Invest Dermatol. 2015;135:229-37.

[37] Bagot M, Porcu P, Ram Wolff C, Khodadoust M, William B, Battistella M, et al. IPH4102, the first-in-class anti-KIR3DL2 mAb, is safe and clinically active in advanced cutaneous T-cell lymphoma (CTCL) patients: results from the dose-escalation part of the IPH4102-101 phase I study. European Organisation for Research and Treatment of Cancer: Cutaneous Lymphoma Task Force; London 2017.

[38] Bagot M, Porcu P, Ram Wolff C, Khodadoust M, Basem W, Battistella M, et al. Phase I study of IPH4102, anti-KIR3DL2 $\mathrm{mAb}$, in relapsed/refractory cutaneous T-cell lymphomas (CTCL): dose-escalation safety, biomarker and clinical activity results. Hematological Oncology. 2017;35(Supplement S2):48-9.

[39] Battistella M, Marie-Cardine A, Paturel C, Bonnafous C, Thonnart N, Khodadoust M, et al. Biomarkers of pharmacological and clinical activity of IPH4102, first-in-class humanized anti-KIR3DL2 mAb, in a phase I study in patients with relapsed/ refractory CTCL. European Organisation for the Research and Treatment of Cancer: Cutaneous Lymphoma Task Force; October 14th; London 2017. 